\title{
Research on Fonts in the Design of Movie Posters
}

\author{
Rui Meng ${ }^{1, *}$ Xiaohui Shen ${ }^{1}$ \\ ${ }^{1}$ Art College, Northeast Electric Power University, Jilin, Jilin 132000, China \\ *Corresponding author. Email: 1260067168@qq.com
}

\begin{abstract}
How to deepen the artistic expression and aesthetic value of fonts in movie posters by adhering to the spirit of national cultural subjects is an important problem that Chinese designers need to solve urgently, and it is also the main purpose of the research. Illustration method and literature research method are used comprehensively in this paper to interpret typical Chinese movie poster works, and then the key role of fonts in accurately conveying movie information in movie posters is drawn out. The forms hidden in the picture, the ink in the brush, and the artistic conception in accordance with the intention can be explored. The combination of such aesthetic implication and technical means such as multidimensional space, diversified texture, dynamic and multi-change not only brings the viewer a strong visual experience, but also endures it with profound cultural connotation, which has both commercial value and artistic value.
\end{abstract}

Keywords: Movie poster, Font design, Aesthetic meaning, Technical application.

\section{INTRODUCTION}

British designer Alan Fletcher emphasized: "The priority in design is how to use design wisdom to create a form of added value and visual surprise [1]. With the appearance and existence of film art, it seems that the boundary between "history" and "art" is disappearing, and the pictures and scenes of "history" are preserved forever. "Art" is such "additional value and visual form surprise". Movie posters, an important subsidiary of the film art, are the form of advertising media that directly display the plot and artistic picture of the film. However, the research finds that "commercial" and "utilitarian" are abundant in the design market of film posters, and there is also a lack of "cultural" and "artistic" and other added values. Therefore, in order to break the homogenization and singleness of the design market, the perceptual structure and artistic interest of the appreciator should be changed. This study analyzes the aesthetic implication of fonts in film poster design from the perspectives of the forms hidden in the picture, the ink in the brush, and the artistic conception in accordance with the intention, and explores the technical application of font design from the dimensions of multidimensional space, diversified texture, dynamic and multi-change, aiming at presenting a unique artistic scene of fonts.

\section{THE AESTHETIC IMPLICATION OF FONT IN MOVIE POSTERS}

In order to further explore the added value of "culture" and "art" brought by fonts in the current poster design, the research mainly discusses the aesthetic implication and cultural connotation of font design from three perspectives: the forms hidden in the picture, the ink in the brush, and the artistic conception in accordance with the intention.

\subsection{The Forms Hidden in the Picture}

Dating back to the prehistoric art period, pictographs were mainly used by people to record important events and communicate with each other. They were the most primitive symbols. In the current design market, the combination of pictures and words in the form of artistic expression is widely used in film poster design. For example, the "black and white" poster of Shadow, the most important element of the whole picture is to present the character "shadow" in running script through the relationship between the picture and the background, and to graphically express the font in an artistic way. The graph representing things appears to be definite, structured, and separated or protruded from a bottomless, shapeless, uniform, unimportant, or often overlooked "bottom" [2]. When staring at the white 
part of the whole, the Chinese character "shadow" is the figure, and "person" is the background. When staring at the local black part, "character" is the figure and the Chinese character "shadow" is the background. With the change of visual focus, "shadow" and "person" are interdependent and interchangeable, reflecting the obscure character relationship and the hidden atmosphere of murder in the film, and bringing viewers a desolate and vigorous visual and auditory feast. The designer's grasp of "picture" and understanding of "bottom" endows the font with interest and the freshness of graphics, which makes the picture more creative and novel. Another example is the poster of Chinese version of the "My Neighbor Totoro", the strokes are steady and thick, the turns rounded and full of strength. Some of the strokes also contain the body and eyes of Totoro, and the replacement relationship between the strokes and the figure makes the soft and pure image of Totoro self-evident. To sum up, designers can not only express content through simple fonts, but also explore an abstract aesthetic sense of form through graphical text and images. An abstract sense of formal beauty can also be explored, through creative thinking to accurately express the designer's deep intention, thought and emotion, directly convey to the viewer's different feelings, and then reflect on the deep cultural connotation.

\subsection{The Ink in the Brush}

In a broad sense, the word "brush and ink" refers to the painting language of picture atmosphere, color, structure, artistic conception and taste achieved by using brush and ink. In a narrow sense, brush and ink specifically refers to the skills of using brush and ink. [2] Contemporary designers have widely used this technique in the form design of film posters to pursue a unique and interesting effect. For example, the "Venezia" version of the poster "Shadow" is designed with the character "Shadow" as the theme of the film. The lines are relaxed and smooth, which not only reflects the vivid portrayal of traditional calligraphy, but also reflects the unique beauty of hieroglyphics. The dry and white brush strokes not only strengthen the flowing sense of the film poster, but also show the pursuit of the clear, elegant and vivid state of the Oriental aesthetic thought. In the traditional painting theory, "ink" can be divided into five shades in detail, namely, coke, thick, heavy, light and clear. The combination of different shades can create a richer sense of color and space. For example, the poster of "Taoist Monk down the Mountain", the free ink and bold and vigorous brush strokes, seem to be in a state of casual writing, or like a carefully designed picture, presenting a visual effect of freehand brushwork. At the same time, the designer uses different shades of ink to represent the surrounding environment, as the harbinger of the little Taoist priest "going up and down" the mountain, which effectively expresses the theme and spiritual connotation of the film. Liu Xizai in the Qing Dynasty gave a new interpretation of "brush and ink" in his book "Yi Qi - Shu Qi". He believed that brush and ink was the unity of "meaning" and "image". Admittedly, in the design of font posters, designers should not only pay attention to the expression of lines and other techniques, but more importantly, be able to carry deep thoughts and special aesthetic taste.

\subsection{The Artistic Conception in Accordance with the Intention}

Artistic conception is not the real situation seen by the eyes, but the painting scene of the spiritual perception from the eyes and the heart. [4] Mr. Zong Baihua believes that through the study of the theory of artistic conception, we can grasp the spirit of Chinese art and explore the connotation of Chinese culture. For this reason, the "artistic conception" of ancient China is common in literary works. For example, in "Drinking Wine" by Tao Yuanming of the Eastern Jin Dynasty: "Picking a chrysanthemum, carefree and leisurely see Nanshan. [5]." With a few strokes, Mr. Tao Qian meets the external image and the internal interest in the countryside, creating a quiet and distant natural artistic conception. Another example is Bai Juyi's "The Journey of Pipa" in the Tang Dynasty: "冰泉冷涩弦凝绝，凝绝不通声暂歇，别有 幽愁暗恨生，此时无声胜有声 [5]"，stimulating the players and listeners sigh "with far up, meet why had known each other", to achieve the effect of "an end to the words, but not to their message". The same approach can be applied to design work. For example, as for the poster of the "Venice" version of the "Shadow", the vertical rotation of the "shadow" occupies one third of the picture, the side part "'ミ" extends, each stroke feels like a step, forming a unique font effect. The ending point is the character in the film, which coincides with the character of Jing Zhou in the film, and also hints at the final outcome of the governor. Brush style and ink style bring out the best in each other, font and graphics are combined into a reasonable layout, enhance the impact of font, create a different artistic conception, and worth exploring and taste. Therefore, font is the key to the theme, aesthetic appeal and artistic atmosphere of the work. It can arouse the audience's artistic association 
and fantasy, so as to achieve the fit of image and taste.

\section{THE TECHNICAL APPLICATION OF FONT IN MOVIE POSTERS}

With the emergence of 3D and VR technology in the film art, the wide application of PS and AI software in graphics, and the diversification and dynamic design of fonts have become the direction and trend of development. The designer closely follows the development of technology, and expresses fonts in multi-dimensional space, diversified texture and dynamic changes, in order to seek innovative and interesting design, so as to attract more viewers' attention and achieve the unity of commercial value and artistic value.

\subsection{Multidimensional Space}

In the initial stage of the development of singledimensional typefaces, the concept of space was limited to the orbit of its linear motion, and typefaces had to have relative position and direction. After the appearance of two-dimensional concept, the basic form of plane composition was rapidly developed, and font design began to change accordingly. As for the evolution and development of the concept of three dimensions, fonts can be arbitrarily extended to infinite space. For example, "The Golden Age" mainly tells the true life of the legendary female writer Xiao Hong in the Republic of China and the fate of her wandering experience, and portrays the true story of a group of hot-hearted young people who fought against the turbulent times with their writing. The design of the film poster is rich and colorful, the most typical being the "pen edition" series of posters. It looks like an architectural design on the whole, transforming the "golden age" of regular style into strong columns and sharp knives by means of deconstruction and isomorphism. The dots are scattered in the poster, seemingly out of order, but actually in order, which enriches the virtual and real effects between the strokes. A small proportion of figures sits quietly or walks in and out of the room, which constitutes a sharp contrast with the brushwork. It constructs a poetic artistic and cultural atmosphere and a complex social and economic background both on the whole and visually, and also presents a writer's unique artistic life on the content. However, they can never escape the fate of being manipulated by the times, and their poems are full of sorrow. It can be seen that the method of deconstruction and isomorphism of basic strokes makes the form of font design more aesthetic, properly expresses the sense of space and volume of strokes, and gives different visual enjoyment to fonts. In addition to disassembling and reorganizing the basic strokes, different design methods, such as perspective, light and shadow, virtual and real, can also be used to create a multi-dimensional space of font design. However, it should be noted that font design is limited by ideology, and the recognition of font should not be affected when the space is multidimensional, which is also one of the basic principles of font design.

\subsection{Diversified Texture}

Historically, Chinese characters developed from inscriptions on bones or tortoise shells of the Shang Dynasty, seal character, clerical script (an ancient style of calligraphy current in the Han Dynasty), cursive script, regular script, to running script, etc. At the same time, writing carriers, such as animal bones, slips, thick bamboo tube, cliffs, and silk manuscripts, are also constantly developing and changing, generating different styles of picture effects. A mature and complete design will not only take into account the visual form of the picture, but also the texture of the picture, so as to achieve a multi-sensory appreciation experience. For example, in the poster font of "The Warlords", the clerical script and the Yin and Yang seals interact with each other. The texture is solemn and simple, calm and restrained, forming a magical restraint between the artificial and the natural, which exactly correspond to the bleak story of love and hate in the film. Another example is the poster font of "Aftershock". The textural design of the character "Aftershock" resonates with the picture of the ruins after the earthquake. The font texture sets off the damage of the earthquake to the people after the disaster, and the sad story of the disaster naturally reveals. In addition, the Chinese version of "My Neighbor Totoro" poster fonts, brush effect and the cute furry image complement each other, letting the viewer feel the taste of nature. Completely different materials and textures show unique design, bringing different visual perceptions. Therefore, in the process of design, designers should fully explore more material carriers, the expression of texture, to meet visitors' demands for aesthetic experience.

\subsection{Dynamics and Multi-change}

Dynamics are also called "motion". Gombrich mentioned in "Sense of Order": "Viewing is selective from the very beginning, and how the eyes respond to the sample depends on many physiological and psychological factors. In order to attract the attention 
of the viewer, the sensory system only detects changes in the distribution of stimuli that can cause new alertness [6]." Gombrich's work shows that, influenced by physiological and psychological factors, visual perception is selective and skipping. It often takes the moving points as the focus of selection and begins to track the movement trajectory of these points. Taking the lettering on the poster for "Let the Bullets Fly" as an example. The fonts in the same arrangement are cut in the same position, and the visual focus moves with the shuttling arrows. The track of the fonts has a sense of speed and extension, which not only corresponds to the story of the fierce fight between Zhang Muzhi and the bullies Huang Silang with unknown results, but also creates infinite imagination space and potential curiosity of the viewers. Another example is the dynamic countdown poster of Lost in Hong Kong. The countdown font pays attention to the visual movement, attracts the visual focus of viewers, and further strengthens the propaganda effect of the film. In short, when creating font posters, designers should take into account the visual trend of viewers and pay attention to the trend of font movement, so that viewers can feel the artistic charm of dynamic font posters through multi-sensory participation. At the same time, font dynamic is also a major development trend in the future.

\section{CONCLUSION}

To sum up, this paper makes a comprehensive analysis of the aesthetic implication of font design from the perspectives of the forms hidden in the picture, the ink in the brush, and the artistic conception in accordance with the intention. The technical application of font design can also be deeply explored from the dimensions of multidimensional space, diversified texture, dynamic and multi-change, so as to seek the added value of "culture" and "art" in movie poster design, carry out organized artistic processing and arrangement of fonts, convey information, and promote consumption. The research results show that when designing movie posters, the first thing to do is to define the design style according to the theme, which requires the designer to fully understand and recognize the theme and plot of the work in the early stage, so as to determine its style more accurately. Secondly, designers need to improve their culture and have a flexible imagination for the use of fonts in movie poster design. Finally, designers need to keep abreast of technological trends to add to the creative development of typography.

\section{AUTHORS' CONTRIBUTIONS}

Rui Meng wrote this paper, and Xiaohui Shen contributed to revising.

\section{REFERENCES}

[1] Wang Shaoqiang. Design Form: Vision. Form - Aesthetics [M]. Guangzhou: Lingnan Fine Arts Publishing House, 2002. (in Chinese)

[2] Rudolf Arnheim. Translated by Teng Shouyao. Visual Thinking-Psychology of Aesthetic Perception [M]. Chengdu: Sichuan People's Publishing House, 2019. (in Chinese)

[3] Qin Mengna. Chinese Painting Culture [M]. Beijing: Current Affairs Publishing House, 2008. (in Chinese)

[4] Guo Chengbo. On the creation of the artistic conception of interior environment design [J]. Hundred Schools in Arts, 2006(03):160-162. (in Chinese)

[5] Zhu Guangqian. Cn Beauty - A Classical Elaboration of Eastern and Western Aesthetics by Zhu Guangqian [M]. Beijing: Jincheng Publishing House, 2006. (in Chinese)

[6] E.H. Gombrich. Translated by Fan Jingzhong. Sense of Order-Psychological Research on Decorative Art [M]. Changsha: Hunan Science and Technology Press, 1999. (in Chinese) 